\title{
Intervalo hídrico ótimo e densidade crítica de um Latossolo Amarelo coeso sob diferentes usos no ecossistema Tabuleiro Costeiro ${ }^{1}$
}

\author{
Camila Brasil Dias ${ }^{2 *}$, Genelício Crusoé Rocha ${ }^{3}$, Igor Rodrigues de Assis ${ }^{3}$, Raphael Bragança Alves Fernandes ${ }^{3}$
}

$10.1590 / 0034-737 X 201663060017$

\begin{abstract}
RESUMO
Os impactos do uso e do manejo na qualidade física do solo têm sido quantificados, utilizando-se o intervalo hídrico ótimo e a densidade crítica do solo. O objetivo deste trabalho foi determinar o intervalo hídrico ótimo (IHO) e a densidade crítica de um Latossolo Amarelo coeso, dos Tabuleiros Costeiros do Recôncavo da Bahia, submetido a diferentes usos e manejo do solo. Foram selecionadas três áreas, sobre solo coeso típico, submetidas aos seguintes usos e manejos: mata nativa (Mata Atlântica); pastagem de Brachiaria decumbens Stapf, em estado de degradação, e cana-de-açúcar, com subsolagem no sulco de plantio. Foram retiradas em cada área 40 amostras com estrutura indeformada, na porção central de cada horizonte (A e AB). Foram determinados nessas amostras, após serem submetidas a dez valores de tensão e pressão, a umidade, a resistência à penetração e a densidade do solo. Na área cultivada com cana, a coleta das amostras foi feita nas linhas de plantio, onde foi realizada a subsolagem. O intervalo hídrico ótimo do horizonte A, da mata, e do Ap, da cana-de-açúcar foram semelhantes e, ambos, maiores que na pastagem. Já no horizonte AB, o IHO da cana-de-açúcar foi maior que o da mata e, o desta, maior que o da pastagem. Os horizontes Ae Ap apresentaram valores de densidade crítica maiores que os de $\mathrm{AB}$ para todos os usos avaliados. O uso que apresentou maior frequência de valores de densidade do solo acima da densidade crítica foi a pastagem.
\end{abstract}

Palavras-chave: qualidade física do solo; resistência à penetração; densidade crítica.

\section{ABSTRACT \\ Least limiting water range and critical density of a cohesive Yellow Oxisol under different land uses in the Tabuleiro Costeiro ecosystem}

The impacts of the use and management on soil physical quality have been quantified using the least limiting water range and the critical density of the soil. The critical soil bulk density obtained by the least limiting water range (LLWR) assists in making decisions on the management conditions adopted or to be adopted in certain soil. This study aimed to determine the LLWR and the critical soil bulk density of a cohesive Oxisoil from Tabuleiros Costeiros of Reconcavo da Bahia subjected to different uses and soil management. We selected three areas on typical cohesive soil, subjected to the following uses and management: native forest (Mata Atlântica), Brachiaria decumbens Stapf, in a state of degradation, and sugar cane, with subsoiling at planting. In each area, 40 samples were taken with undisturbed structure in the central portion of each horizon (A and AB). In the area cultivated with sugarcane, the sample collection was done in the rows. The LLWR in the A horizon of the forest and the sugar cane were similar and both were greater than the pasture on the horizon AB. The LLWR for cane sugar was higher than the forest and this was higher than the pasture. Horizons A and Ap presented higher critical density values than AB for all evaluated uses. The use that showed values of bulk density greater than the critical density was pasture.

Key words: soil physical quality; penetration resistance; critical density.

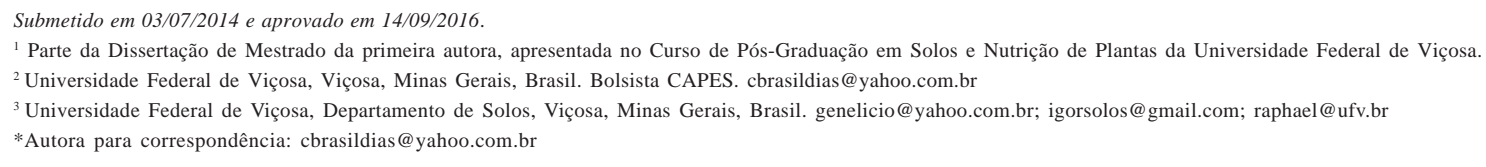




\section{INTRODUÇÃO}

Os Tabuleiros Costeiros são formações terciárias que se distribuem por quase toda a faixa costeira do Brasil, desde o Estado do Amapá até o Rio de Janeiro, e cujos solos, apesar de profundos e situados em relevo plano, apresentam limitações à produção vegetal (Souza, 1996; Melo Filho et al. 2004; Souza, 2005). Estima-se que, no Brasil, as áreas de Tabuleiros cheguem a 20 milhões de hectares, sendo que, destes, nove a dez milhões encontram-se na região nordeste, constituindo a principal base de sustentação agrícola dos estados e capitais da costa oriental do Brasil (Souza, 2005).

Nessa grande unidade de paisagem, os principais solos (Latossolos Amarelos coesos e Argissolos Amarelos coesos) caracterizam-se como profundos, ácidos, com baixa capacidade de troca catiônica e apresentam frequentemente horizontes coesos (duros) (Rezende, 2000). Corrêa et al. (2008) sugeriram que a gênese de horizontes coesos devese ao maior conteúdo de argila muito fina $(<0,2 \mu \mathrm{m})$, translocada entre os horizontes, ou dentro do mesmo horizonte, na forma de argila dispersa. Já Moreau (2006) obteve as mesmas conclusões apresentadas por Corrêa et al. (2008) para explicar a formação de horizontes coesos de Argissolos, localizados em platôs mais amplos e menos dissecados, não dando ênfase ao tamanho da argila. Recentemente, Lima Neto et al. (2010) sugeriram que a gênese do caráter coeso apresenta duas fases distintas, sendo formado inicialmente pelo entupimento dos poros decorrentes da iluviação de argila fina, havendo posteriormente a perda de Fe na parte superior, que colapsa a estrutura e provoca o ajuste face a face da caulinita.

Considerando-se as limitações agrícolas dos solos coesos, dos Tabuleiros Costeiros, qualquer interferência de uso e de manejo desses solos, no sentido de aumentar a produtividade das culturas, deverá passar necessariamente pela melhoria da qualidade física do solo e de suas condições químicas.

A qualidade física do solo para o crescimento das plantas é determinada não só pela disponibilidade de água, aeração e temperatura, mas também pela resistência que a matriz do solo oferece à penetração das raízes (Hamblin, 1985; Letey, 1985). Num solo degradado, além da redução da quantidade de água disponível, a taxa de difusão de oxigênio e a resistência do solo à penetração podem limitar o crescimento das plantas na faixa de potenciais que determina a disponibilidade de água no solo (Araujo et al. 2004). Dessa maneira, a caracterização dos efeitos dos sistemas de uso e de manejo sobre a degradação da qualidade física do solo é mais bem quantificada por medidas integradoras dessas modificações.

Vários atributos podem ser utilizados para caracterizar e qualificar a estrutura do solo, mas a resposta das plantas dependerá da interação entre eles (Reichert et al. 2003). Desse modo, torna-se difícil estabelecer de maneira isolada o efeito desses atributos sobre o crescimento das plantas. Com o objetivo de integrar as principais propriedades físicas do solo determinantes do crescimento das plantas, Silva et al. (1994) propuseram o intervalo hídrico ótimo (IHO) do solo, baseado nos pressupostos de Letey (1985), como um índice para avaliar a qualidade física do solo.

De acordo com Kaiser et al. (2009), o IHO pode ser considerado um "semáforo" da qualidade estrutural do solo. As condições de umidade ideais, para o desenvolvimento e crescimento das plantas, ocorrem entre os limites superior e inferior do IHO. As condições são limitantes quando se situam acima ou abaixo dos limites do IHO e são críticas para o crescimento das plantas quando a densidade do solo estiver acima da densidade em que o IHO é nulo (Silva \& Kay, 1996, 1997; Collares et al. 2006). Adensidade crítica obtida por meio do IHO auxilia na tomada de decisões diante das condições de manejo adotadas ou a serem adotadas em determinado solo.

Diante do exposto, o objetivo deste trabalho foi determinar o IHO e a densidade crítica de um Latossolo Amarelo coeso, dos Tabuleiros Costeiros do Recôncavo da Bahia, submetido a diferentes usos e manejo do solo.

\section{MATERIAL E MÉTODOS}

No campus da Universidade Federal do Recôncavo da Bahia (UFRB), localizado no município de Cruz das Almas, foram selecionadas três áreas, sobre solo coeso típico, submetidas aos seguintes usos e manejos: mata nativa (Mata Atlântica); pastagem de Brachiaria decumbens Stapf, em estado de degradação, e cana-de-açúcar, com subsolagem no sulco de plantio. O solo, característico da grande unidade de paisagem Tabuleiros Costeiros (Silva et al., 1993) foi classificado como Latossolo Amarelo distrófico coeso, relevo plano, textura média, originário do sedimento Grupo Barreiras (Embrapa, 1999). A composição granulométrica e a classe textural são apresentadas na Tabela 1.

O cultivo da cana-de-açúcar foi implantado em uma área anteriormente ocupada com pastagem, em estado de degradação, pois apresentava perda de vigor, típicos sinais de deficiência de nutrientes, invasão de ervas daninhas, além de elevada resistência à penetração. Com o objetivo de reduzir a elevada resistência à penetração, a área foi subsolada, em agosto de 2009, com subsolador de três hastes distanciadas entre si de $0,50 \mathrm{~m}$ e com profundidade efetiva de trabalho de aproximadamente $0,45 \mathrm{~m}$. A cana foi plantada no sulco de subsolagem.

Em cada trincheira foram retiradas 40 amostras com estrutura indeformada, na porção central de cada horizonte, totalizando 240 amostras coletadas nas três áreas. Para 
a retirada dessas amostras utilizou-se um amostrador de solos modelo Uhland, com cilindros de dimensões de $5 \mathrm{~cm}$ de altura e $5 \mathrm{~cm}$ de diâmetro. Na área cultivada com cana, a coleta das amostras foi feita nas linhas de plantio, em que foi realizada a subsolagem. Após a coleta, as amostras foram cuidadosamente revestidas com filme de PVC e acondicionadas em caixa de isopor para serem encaminhada para laboratório.

Após devidamente preparadas, as amostras com estrutura indeformada foram saturadas, durante 48 horas, por elevação gradual de lâmina de água numa bandeja, até atingir cerca de $2 / 3$ da altura da amostra.

Para obtenção das curvas de retenção de água no solo (CRA), as amostras foram submetidas aos seguintes potenciais mátricos: na mesa de tensão, $-0,004,-0,006,-0,008$ e -0,01 MPa; nas câmaras de Richards, -0,03, -0,05, -0,07, 0,1,-0,5 e -1,5 MPa, de acordo com o que recomenda Silva et al. (1994). Foram realizadas quatro repetições para cada valor de potencial mátrico. Todas as CRAs foram posteriormente ajustadas pelo modelo proposto por Van Genuchten (1980), indicado na equação 1:

$\theta=\theta r+(\theta s-\theta r) /\left[1+(\alpha \psi)^{n}\right]^{m}$

em que:

$\theta=$ Umidade do solo $\left(\mathrm{g} \mathrm{g}^{-1}\right)$;

$\theta \mathrm{r}=$ Umidade residual do solo (na tensão de 1,5 MPa), $(\mathrm{g}$ $\left.\mathrm{g}^{-1}\right)$;

$\theta \mathrm{s}=$ Umidade do solo saturado $\left(\mathrm{g} \mathrm{g}^{-1}\right)$;

$\psi=$ Potencial da água no solo $(\mathrm{kPa})$;

$\alpha, \mathrm{m}$ e $\mathrm{n}=$ parâmetros empíricos da equação obtidos pelo ajuste do modelo.

Os parâmetros $\alpha, \mathrm{m}, \mathrm{n}$ e os valores de $\theta$ s e $\theta$ r foram estimados, utilizando-se o software Soil Water Retention Curve (SWRC versão 3.00), desenvolvido por Dourado Neto et al. (2001).

Após atingido o equilíbrio (quando toda a água presente na amostra de solo com energia de ligação com o solo menor que a energia aplicada é retirada), para cada valor de potencial mátrico aplicado, as amostras foram submetidas à análise de resistência mecânica do solo à penetração (RP), em penetrômetro de bancada, com velocidade de penetração constante de $1 \mathrm{~cm} \mathrm{~min}^{-1} \mathrm{e}$, em seguida, colocadas para secar em estufa, a $105^{\circ} \mathrm{C}$, por $48 \mathrm{~h}$, para determinação da densidade do solo, de acordo com Embrapa (2011). Assim, utilizaram-se as mesmas amostras indeformadas selecionadas para a determinação das curvas características de retenção da água no solo, submetidas aos mesmos potenciais mátricos indicados anteriormente.

As medidas de RP obtidas nas camadas compreendidas entre 0 e 1,0 cm e entre 4 e $5 \mathrm{~cm}$ de profundidade foram descartadas, uma vez que essa resistência aumenta até determinada profundidade e depois tende a tornar-se constante. Bradford (1986) definem-na como profundidade crítica de penetração. Sendo assim, considerou-se a média dos valores de resistência à penetração observada na camada de 1 a 4 cm de cada amostra.

A resistência à penetração é influenciada pela densidade do solo e pelo conteúdo de água do solo $(\theta)$, de forma que uma relação funcional entre estes atributos pode ser identificada como curva de resistência do solo (CRS). A CRS foi ajustada por meio de um modelo não linear, proposto por Busscher (1990), conforme equação 2.

$R P=a \theta^{b} \cdot D s^{c}$

Os valores de $\theta$ foram ajustados em relação à densidade do solo (Ds) e ao potencial da água no solo $(\psi)$, utilizando-se um modelo de regressão não linear, proposto por Tormena et al. (1998), conforme equação 3.

$\theta=\exp (d+e \cdot D s) \cdot(\psi)^{f}$

em que:

a,b,c,d,e,f = parâmetros de ajuste;

$\theta=$ é a umidade do solo;

Ds = é a densidade do solo;

$\psi=$ é o potencial da água no solo.

Para a construção do diagrama do IHO a partir das equações 4, 5, 6 e 7, geradas com base nas equações 4 e 5,

Tabela 1: Composição granulométrica, argila dispersa em água (ADA), grau de floculação (GF), densidade de partículas do solo (Dp) e classe textural de um Latossolo Amarelo coeso, dos Tabuleiros Costeiros do Recôncavo da Bahia, submetido a diferentes manejos

\begin{tabular}{|c|c|c|c|c|c|c|c|c|c|}
\hline \multirow{2}{*}{ Uso } & \multirow{2}{*}{ Horiz. } & \multirow{2}{*}{$\mathbf{A G}^{*}$} & $\mathbf{A F}^{* *}$ & Silte & Argila & \multirow{2}{*}{ ADA } & GF & Dp & \multirow{2}{*}{ ClasseTextural } \\
\hline & & & & $\mathrm{g} \mathrm{kg}^{-1}$ & & & $\%$ & $\mathrm{~kg} \mathrm{dm}^{-3}$ & \\
\hline Mata & $\mathrm{A}$ & 582,5 & 182,5 & 17,3 & 200,0 & 45,7 & 77,15 & 2,66 & Franco argiloarenoso \\
\hline Mata & $\mathrm{AB}$ & 552,8 & 199,1 & 13,8 & 234,0 & 88,6 & 62,13 & 2,66 & Franco argiloarenoso \\
\hline Pasto & Ap & 599,4 & 203,2 & 17,7 & 179,7 & 57,8 & 67,83 & 2,69 & Franco-arenoso \\
\hline Pasto & $\mathrm{AB}$ & 511,3 & 213,3 & 16,4 & 259,1 & 103,1 & 60,20 & 2,73 & Franco argiloarenoso \\
\hline Cana & Ap & 477,6 & 178,6 & 26,7 & 256,9 & 87,0 & 59,35 & 2,74 & Franco argiloarenoso \\
\hline Cana & $\mathrm{AB}$ & 442,8 & 185,2 & 28,4 & 298,2 & 112,5 & 62,27 & 2,69 & Franco argiloarenoso \\
\hline
\end{tabular}

$* \mathrm{AG}=$ areia grossa; $* * \mathrm{AF}$ areia fina.

Rev. Ceres, Viçosa, v. 63, n.6, p. 868-878, nov/dez, 2016 
utilizou-se algoritmo desenvolvido em EXCEL (Leão \& Silva, 2004).

$$
\begin{aligned}
& \theta=\exp (e \cdot D s) \cdot 0,01^{f} \\
& \theta=\exp (e \cdot D s) \cdot 0,15^{f} \\
& \theta_{R P}=\left(2 / a D s^{c}\right)^{1 / 2} \\
& \theta_{A P}=(1-D s / D p)-0,1
\end{aligned}
$$

Neste diagrama, relacionam-se densidade do solo (Ds) com a umidade na capacidade de campo $\left(\theta_{\mathrm{CC}}\right)$ - ou conteúdo de água no potencial de -0,01 MPa (Haise et al., 1955); com o ponto de murcha permanente $\left(\theta_{\mathrm{PMP}}\right)$ - ou conteúdo de água no potencial de -1,5 MPa (Richards \& Weaver, 1944; Savage et al., 1996); com o conteúdo de água no solo $\left(\theta_{\mathrm{PA}}\right)$, em que a porosidade de aeração é de $0,10 \mathrm{~m}^{3} \mathrm{~m}^{-3}$ (Grable \& Siemer, 1968), e com o conteúdo de água no solo $\left(\theta_{\mathrm{RP}}\right)$, em que a resistência mecânica do solo à penetração atinge o limite crítico de 2,0 MPa (Taylor et al., 1966). Os valores de $\theta_{\mathrm{RP}}$ foram obtidos por meio do modelo matemático ajustado aos dados de resistência. $O$ valor de $\theta_{\mathrm{PA}}$, em que a porosidade de aeração é de $0,10 \mathrm{~m}^{3} \mathrm{~m}^{-3}$, foi obtido por meio da expressão [(1-Ds/ Dp) - 0,1]. Foi considerado um valor de Dp médio por horizonte, em $\mathrm{kg} \mathrm{dm}^{-3}$, como sendo a densidade média de partículas (Dp) neste solo, conforme determinações da Embrapa (1997).

O IHO foi calculado como a diferença entre os limites superior e inferior dos conteúdos de água em que ocorrem os parâmetros físicos considerados limitantes. O limite superior é o menor valor de $\theta$ considerado na capacidade de campo, ou na porosidade com aeração mínima de $10 \%$, e o limite inferior é o maior valor de $\theta$ para resistência à penetração máxima de 2,0 MPa ou no ponto de murcha permanente.

\section{RESULTADOS E DISCUSSÃO \\ Intervalo Hídrico Ótimo (IHO)}

\section{Horizonte A (mata) e Ap (solos cultivados):}

O IHO para os horizontes A e Ap do solo sob os diferentes usos (Figura 1), representado pela área hachurada, mostra a grande amplitude de umidade do solo em que são mínimas as limitações ao desenvolvimento das plantas. Segundo Tormena et al. (1999) e Silva et al. (2011), quando a $\theta_{\mathrm{PA}}>\theta_{\mathrm{CC}}$ (o limite superior do IHO é a $\theta_{\mathrm{CC}}$ ) em solos com altos valores de densidade, ocorre uma microestrutura estável do solo que preserva o espaço poroso necessário para a troca de gases.

A resistência à penetração para os solos estudados foi o fator que limitou o IHO para os usos com cana e pastagem. Na mata, a RP foi limitante para valores de densidade do solo maiores que $1,36 \mathrm{~kg} \mathrm{dm}^{-3}$. Esses resultados estão de acordo com os obtidos por Topp et al. (1994) e Silva et al. (1994), nos quais a resistência foi o fator que mais redu- ziu o IHO em solos sob diferentes condições de textura e de manejo. A constatação da RP como principal fator físico limitante às plantas também foi verificada por Silva et al. (2009) e Bengough et al. (2011), visto que a variação de RP é dependente da variação de $\theta$ e influenciada pelo aumento de Ds.

Os resultados de IHO do horizonte Ap, obtidos no solo sob mata, foram similares aos do solo sob cana-de-açúcar (subsolada) e ambos apresentaram maiores valores de IHO que o solo sob pastagem. Esses resultados evidenciam que a degradação da estrutura do solo pelo cultivo de pastagem impõe limitações ao crescimento das plantas pela elevada densidade do solo e pela elevada resistência do solo à penetração, que pode atingir valores altos, principalmente em condições de solo com baixa umidade. Essas constatações têm um significado especial para o manejo dos solos coesos, pois, além de a RP atuar como fator limitante ao crescimento do sistema radicular, ela supera o valor de 2,0 MPa, mesmo em altos valores de umidade volumétrica.

Também vale ressaltar que nos três usos avaliados (com exceção ao da mata para valores de Ds menores que $1,36 \mathrm{~kg} \mathrm{dm}^{-3}$ ), o IHO foi menor que a capacidade de água disponível $\left(\mathrm{CAD}=\theta_{\mathrm{CC}}-\theta_{\mathrm{PMP}}\right)$, o que caracteriza os solos como fisicamente limitantes (Letey, 1985). Por esse motivo, Silva et al. (1994) apontam o IHO como uma ferramenta de análise da estrutura do solo mais sensível que o conceito de capacidade de água disponível.

\section{Horizonte $A B$}

Assim como nos horizontes A e Ap, para os três usos avaliados, o limite superior do IHO no horizonte $\mathrm{AB}$ foi a umidade na capacidade de campo $\left(\theta_{\mathrm{CC}}\right)$, indicando que as plantas não terão seu desenvolvimento limitado pela reduzida difusão de oxigênio no solo (Figura 2). Os limites inferiores do IHO para a mata e cana foram a umidade no ponto de murcha permanente $\left(\theta_{\mathrm{PMP}}\right)$ e a resistência do solo à penetração $\left(\theta_{\mathrm{RP}}\right)$. A $\theta_{\mathrm{PMP}}$ passa a ser limitante, nos solos sob mata e sob cana-de açúcar (com subsolagem), para valores de densidades abaixo de 1,40 e $1,45 \mathrm{~kg} \mathrm{dm}^{-3}$, respectivamente.

O IHO do horizonte AB do solo sob pastagem foi limitado pela $\theta_{\mathrm{RP}}$ em $100 \%$ das amostras, ou seja, esse solo apresenta limitações físicas ao desenvolvimento radicular.

Nessa área, o impacto da RP sobre o limite inferior do IHO foi maior, em decorrência da forte relação da densidade do solo sobre a resistência à penetração. Esse resultado é típico de solos adensados ou compactados, que necessitam estar com umidade elevada para que a RP não atinja o valor limitante. Diversos autores também verificaram a influência da $\theta_{\mathrm{RP}}$ para o IHO do solo sob diferentes sistemas de manejo (Leão et al. 2004; Beutler et al. 2006; Silva et al., 2008; Pereira, 2010; Pereira et al. 2012), e para solos com diferentes texturas (Silva et al. 1994; Tormena et al. 1998). 

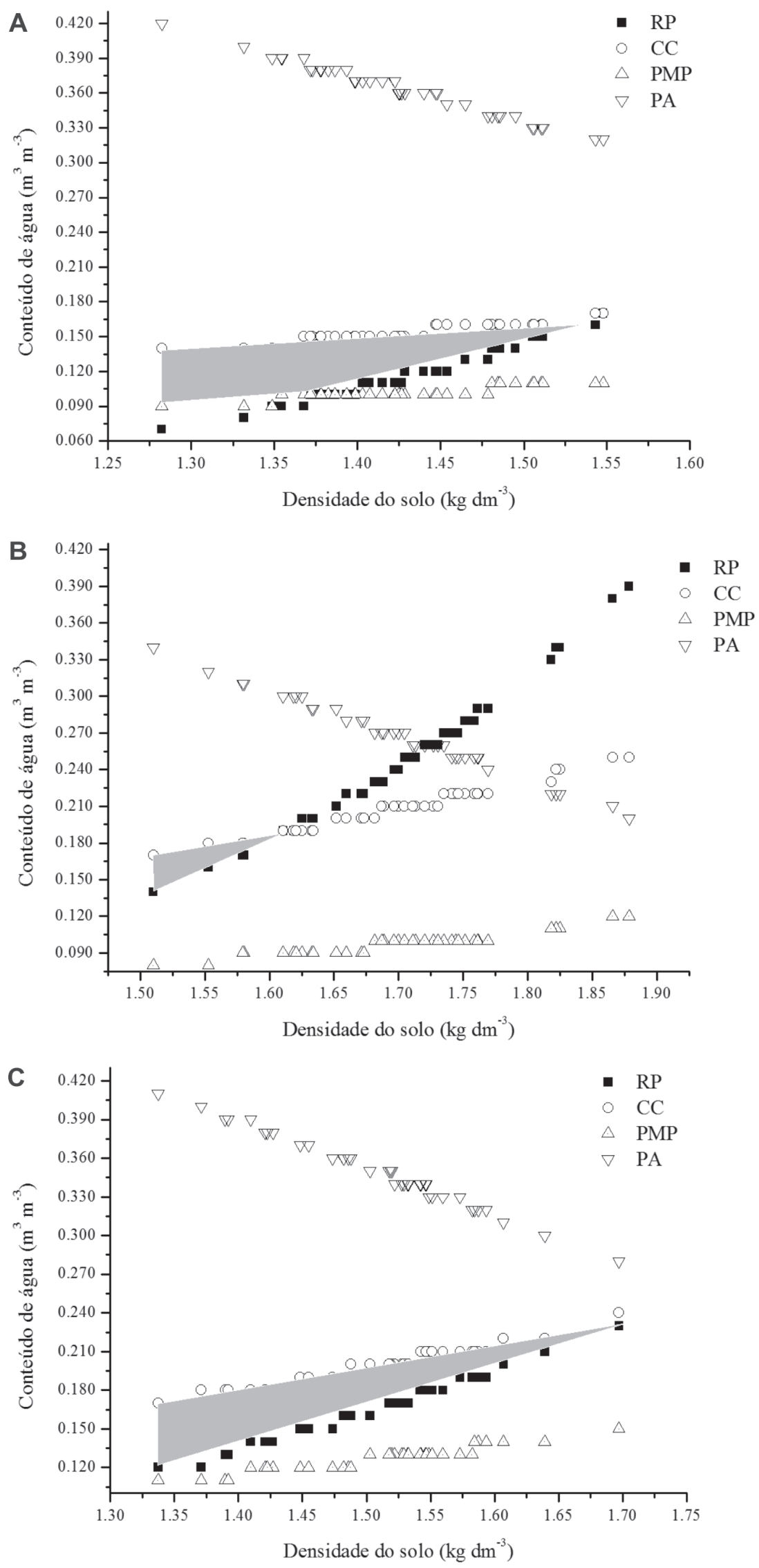

Figura 1: Variação do conteúdo de água com a Ds nos níveis críticos da capacidade de campo $(\psi=-0,01 \mathrm{MPa})$, ponto de murcha permanente $(\psi=-1,5 \mathrm{MPa})$, porosidade de aeração de $10 \%$ e resistência à penetração de $2 \mathrm{MPa}$, no Latossolo Amarelo coeso sob (A) mata (horiz. A); (B) pasto e (C) cana-de-açúcar (horizonte Ap). A área cinza representa o IHO do solo. 

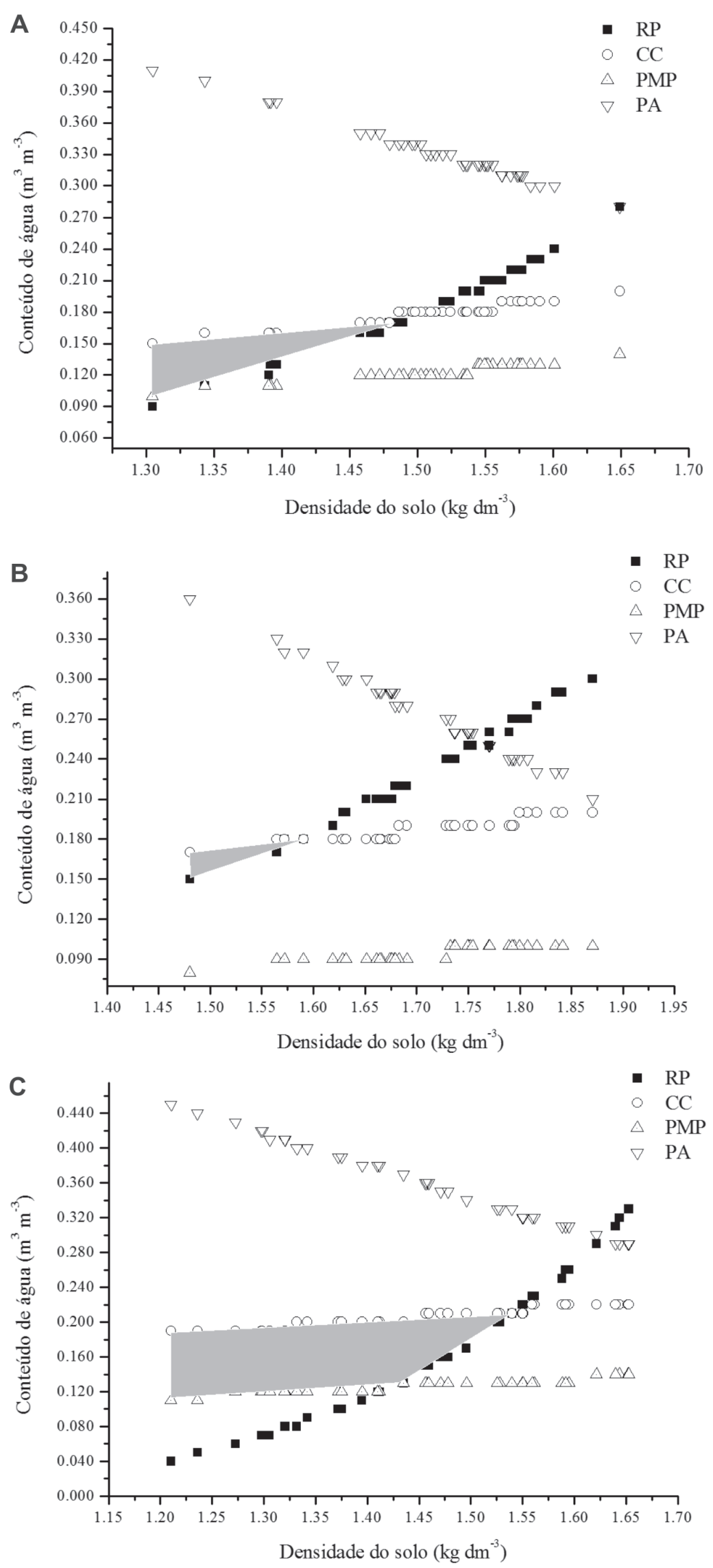

Figura 2: Variação do conteúdo de água com a Ds nos níveis críticos da capacidade de campo ( $\psi=-0,01 \mathrm{MPa})$, ponto de murcha permanente $(\psi=-1,5 \mathrm{MPa})$, porosidade de aeração de $10 \%$ e resistência à penetração de $2 \mathrm{MPa}$, no Latossolo Amarelo coeso, sob (A) mata; (B) pasto e (C) cana-de-açúcar, todos no horizonte AB. A área cinza representa o IHO do solo. 
À medida que houve aumento dos valores de densidade do solo o IHO reduziu-se em todos os usos avaliados, pois ocorreu aumento acentuado da resistência à penetração com a menor variação da umidade do solo. Este efeito, não comum em outras classes de solo, possivelmente, decorre do caráter coeso desse solo.

Nos horizontes A e AB, respectivamente, as amplitudes do IHO do solo nos diferentes usos apresentaram a seguinte ordem: cana-de-açúcar (subsolada) 0,05 e $0,08 \mathrm{~m}^{3} \mathrm{~m}^{-3}$ > mata 0,05 e $0,05 \mathrm{~m}^{3} \mathrm{~m}^{-3}>$ pastagem 0,03 e $0,02 \mathrm{~m}^{3} \mathrm{~m}^{-3}$. $\mathrm{O}$ rompimento da camada coesa pela subsolagem no solo sob cana-de-açúcar modificou o comportamento físico do solo
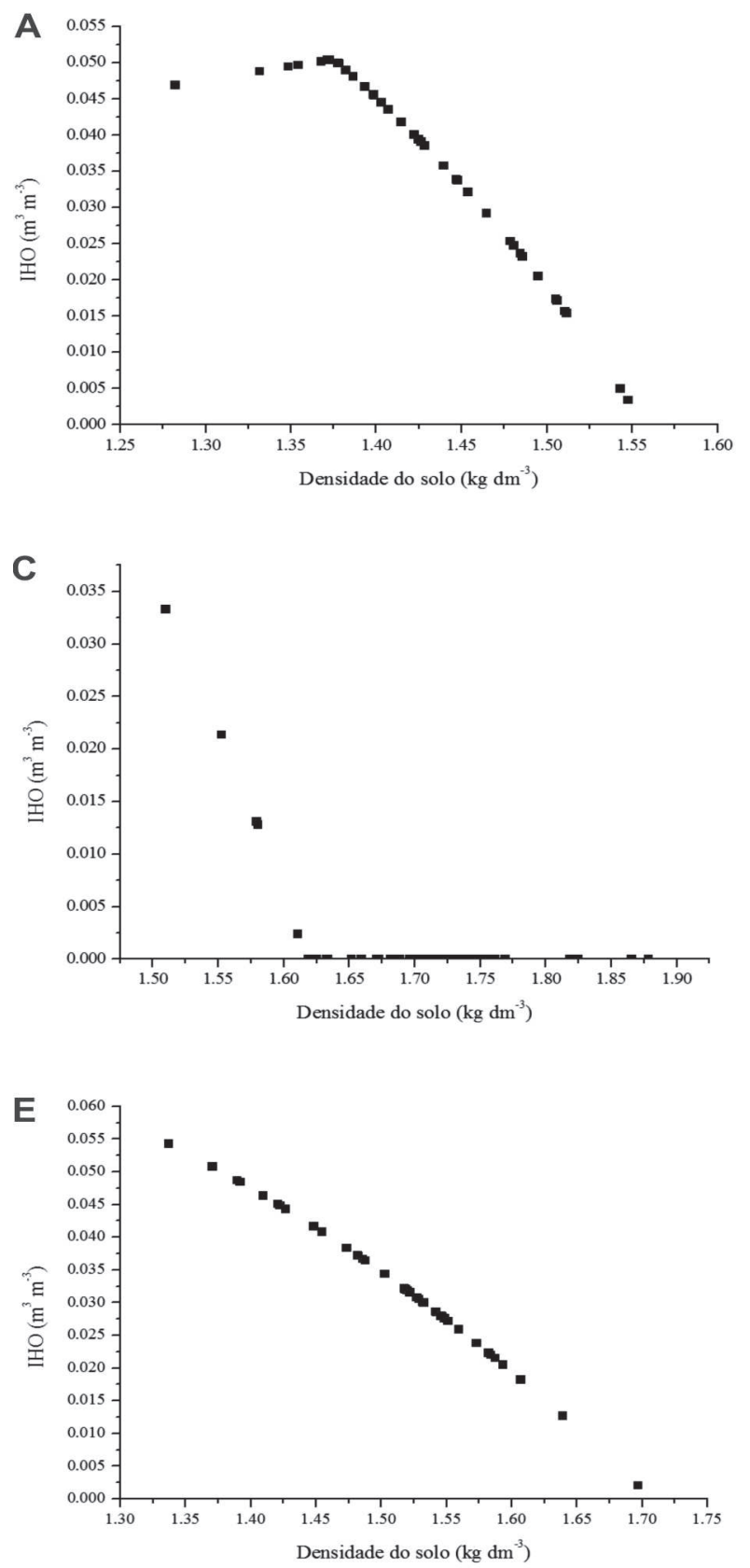

Figura 3: Variação do IHO em função da densidade do solo (Ds) no Latossolo coeso sob (A) mata (horiz. A); (B) mata (horiz. AB); (C) pasto (horiz. Ap); (D) pasto (horiz. AB); (E) cana-de-açúcar (horiz. Ap) e (F) cana-de-açúcar (horiz. AB).

Rev. Ceres, Viçosa, v. 63, n.6, p. 868-878, nov/dez, 2016 
resistência à penetração a que as culturas são sujeitas principalmente nos períodos de veranicos.

Os valores de IHO, em função da densidade do solo são apresentadas na Figura 3. Os resultados mostram a influência negativa da densidade do solo, o que está de acordo com os resultados de Silva et al. (1994), Leão et al. (2005), Medeiros et al. (2011), Silva et al. (2011).

Os resultados também evidenciam que, comparado com os horizontes Ae Ap, o horizonte AB impõe maiores limitações ao crescimento das plantas sob elevada Ds e resistência do solo à penetração, por causa da natureza coesa, que são reduzidas com a subsolagem.
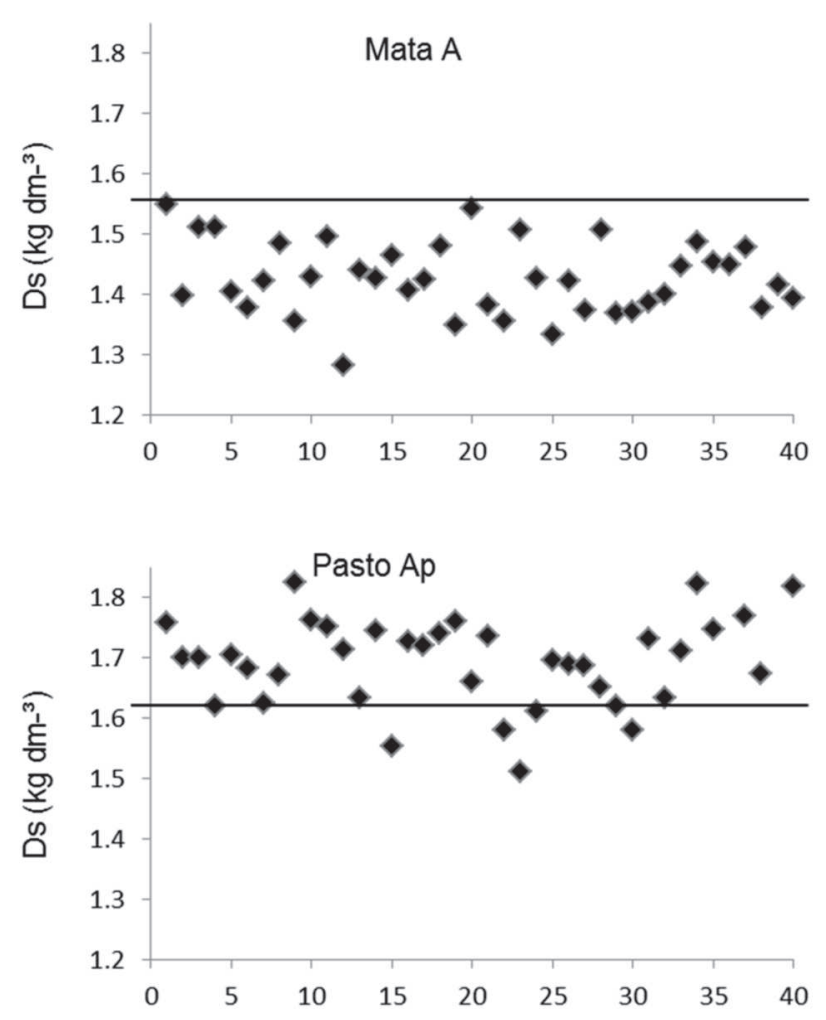

Amostra do solo

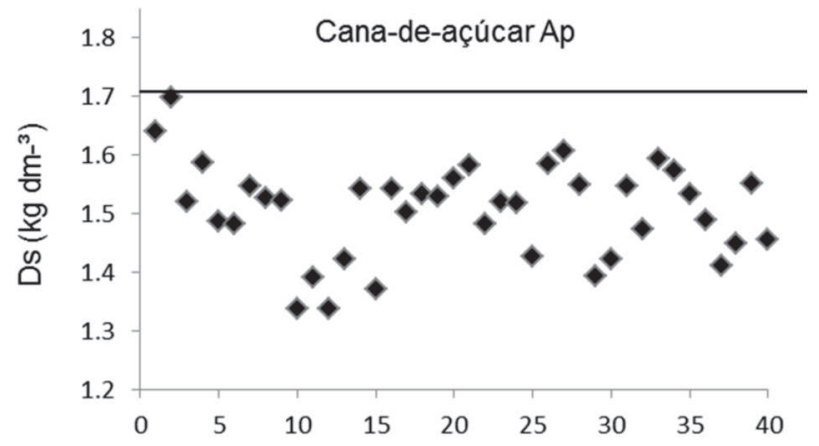

Considerando-se a área de mata como referência, é possível afirmar que o uso com a pastagem provocou degradação do solo, resultando na menor amplitude do IHO, pois os estreitos valores do IHO, tanto para o horizonte Ap quanto para o horizonte $\mathrm{AB}$, indicam que a pastagem está submetida a restrições por excessiva impedância mecânica (elevada RP), principalmente no período de estiagem, conforme teorizado por Kay (1989).

A amplitude dos dados de IHO do solo sob cana-deaçúcar em comparação com a dos dados dos solos sob a mata e sob pastagem mostra o efeito positivo da subsolagem, que, ao romper a camada coesa, promoveu
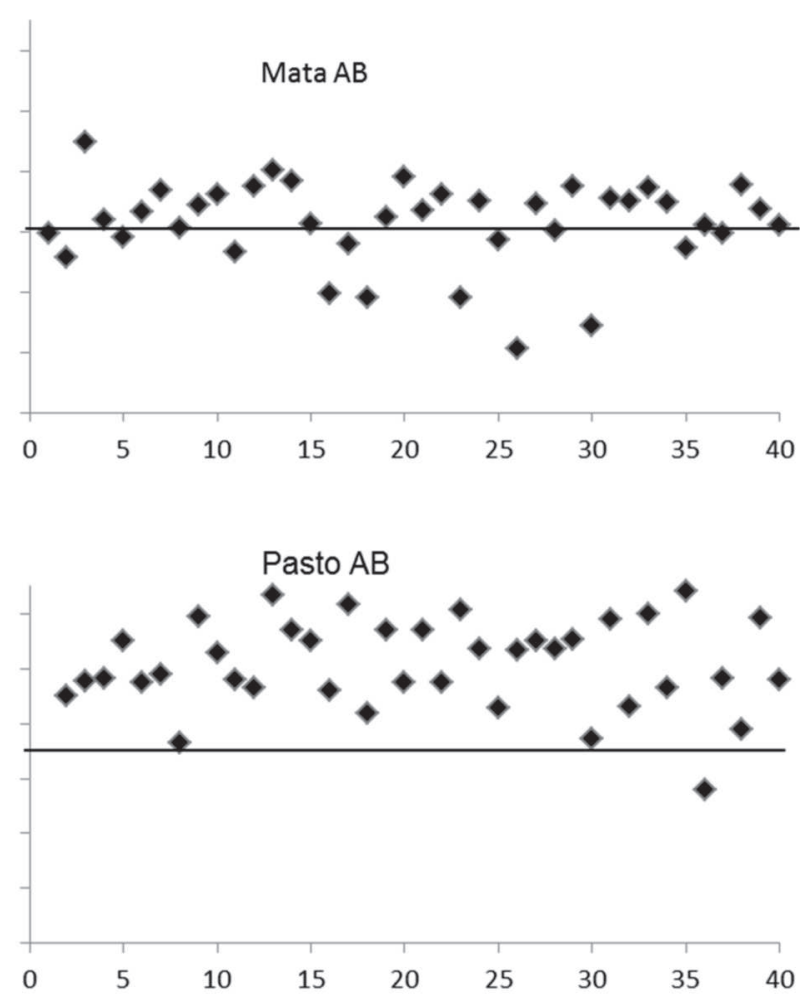

Amostra do solo

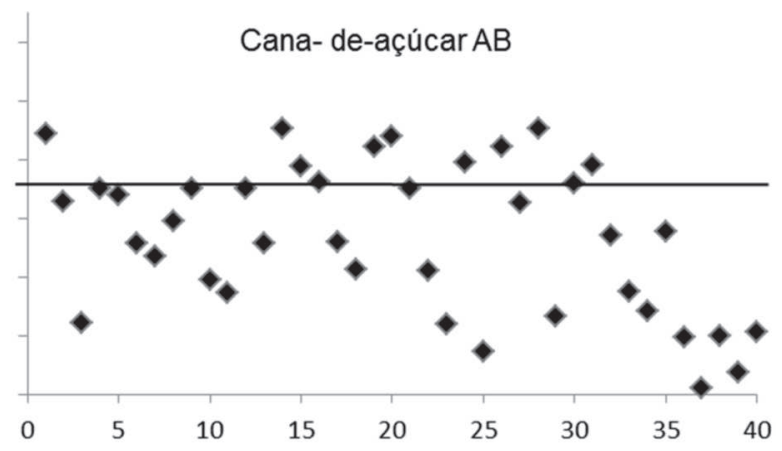

Amostra de solo

Figura 4: Densidade do solo de amostras coletadas nos horizontes A, Ap e AB, em comparação com a densidade crítica do solo (Dsc) para um Latossolo Amarelo distrófico coeso, sob diferentes usos. A linha horizontal indica a Dsc. 
maior amplitude do IHO para o solo, principalmente no horizonte $\mathrm{AB}$ (mesmo três anos após sua realização), o que pode ser explicado pela redução da RP e a redistribuição da porosidade.

\section{Densidade Crítica (Dsc)}

A Dsc é a densidade do solo em que o IHO iguala-se a zero, ou seja, em que o limite inferior de água no solo iguala-se ao limite superior (Silva et al., 1994). A Tabela 2 mostra os valores de Dsc para as diferentes áreas e horizontes estudados. Com o aumento da densidade do solo, para as três áreas, houve diminuição do IHO, até que este atingisse sua densidade crítica. Neste estudo, os horizontes superficiais (A e Ap) apresentaram valores de Dsc maiores que os de $\mathrm{AB}$, tendência associada ao adensamento natural que o horizonte subsuperficial apresenta.

Entre os usos do solo avaliados, verifica-se que o solo sob pastagem foi o que mais apresentou valores de Ds > Dsc. No solo sob mata e no sob cana-de-açúcar, os números de amostras do solo com Ds < Dsc foram semelhantes para o horizonte A e Ap. (Figura 4). Esses resultados indicam que a maior parte da área de pastagem encontrava-se com condições físicas desfavoráveis ao crescimento das plantas, ao contrário dos solos sob mata e sob cana-deaçúcar, subsolado.

Segundo Silva \& Kay (1997) e Leão et al. (2004), limitações ao crescimento radicular podem ocorrer com valores de Ds < Dsc, quando o IHO é muito estreito. Este é o caso do solo sob pastagem, no qual a resistência do solo à penetração tem aumento acentuado à medida que a umidade do solo fica abaixo da capacidade de campo. Isso indica que, mesmo o solo apresentando Ds < Dsc, sua RP pode restringir o crescimento radicular das plantas, inclusive antes de a umidade do solo ficar próxima à do ponto de murcha permanente.

Os altos valores de Dsc observados podem ser explicados pelos elevados valores de Ds, por causa da textura e da camada coesa que o solo apresenta. É comum observar valores de Ds > Dsc, porém existe uma tendência, nos trabalhos de IHO, de que solos sob sistemas de manejo intensivos apresentem, com mais frequência, valores de Ds > Dsc (Araujo et al., 2004; Leão et al., 2006; Tormena et al., 2008). Utilizando-se o conteúdo de argila como referência, Reinert et al. (2001) propuseram valores críticos de

Tabela 2: Valores de densidade crítica do solo relacionados com as áreas e os horizontes estudados

\begin{tabular}{lcc}
\hline \multirow{2}{*}{ Uso } & \multicolumn{2}{c}{ Dsc $\left(\mathbf{k g ~ d m}^{-3}\right)$} \\
\cline { 2 - 3 } & Horizontes A e Ap & Horizonte AB \\
\hline Mata & 1,54 & 1,50 \\
Pasto & 1,61 & 1,56 \\
Cana & 1,70 & 1,55 \\
\hline
\end{tabular}

densidade do solo, de $1,45 \mathrm{~kg} \mathrm{dm}^{-3}$, para solos com mais de $55 \%$ de argila, de $1,55 \mathrm{~kg} \mathrm{dm}^{-3}$, para solos com teor de argila entre 20 e $55 \%$, e de $1,65 \mathrm{~kg} \mathrm{dm}^{-3}$, para solos com menos de $20 \%$ de argila.

Araujo et al (2004) observaram valores de Ds > Dsc para um solo sob cultivos anuais e nenhum valor de Ds $>$ Dsc foi observado para o mesmo solo sob vegetação natural. Já Tormena et al. (1998) obtiveram Dsc de 1,28 kg $\mathrm{dm}^{-3}$, em Latossolo Vermelho, com $800 \mathrm{~kg} \mathrm{dm}^{-3}$ de argila na camada de 0 a $10 \mathrm{~cm}$. Cavalieri et al. (2006) também observaram maior Dsc em tratamentos, envolvendo solos manejados mecanicamente, reduzindo-se a probabilidade de ocorrência de amostras com densidades superiores à Dsc.

\section{CONCLUSÕES}

Os intervalos hídricos ótimos nos horizontes A da mata e Ap da cana-de-açúcar foram semelhantes, e, ambos, maiores que na pastagem. Já no horizonte AB o IHO para a cana-de-açúcar foi maior que para a mata e este maior que para a pastagem.

A amplitude dos dados de IHO do solo sob cana-deaçúcar em comparação com a dos solos sob mata e sob pastagem mostra o efeito positivo da subsolagem, que, ao romper a camada coesa, promoveu maior amplitude do IHO para o solo, principalmente no horizonte $\mathrm{AB}$ (mesmo três anos após sua realização).

Os horizontes A e Ap apresentaram maiores valores de densidade crítica que o horizonte $\mathrm{AB}$, para todos os usos avaliados. $\mathrm{O}$ uso que apresentou maior frequência de valores de densidade do solo acima da densidade crítica foi a pastagem.

\section{REFERÊNCIAS}

Araujo MA, Tormena CA, Inoue TT \& Costa ACS (2004) Efeitos da escarificação na qualidade física de um Latossolo Vermelho distroférrico após treze anos de semeadura direta. Revista Brasileira de Ciência do Solo, 28:459-504.

Beutler AN, Centurion JF, Silva AP \& Barbosa JC (2006) Intervalo hídrico ótimo e produtividade de cultivares de soja. Revista Brasileira de Engenharia Agrícola e Ambiental, 10:639-645.

Bengough AG, Mcckenzie BM, Hallett PD \& Valentine TA (2011) Root elongation, water stress, and mechanical impedance: A review of limiting stresses and beneficial root tip traits. Journal of Experimental Botany, 62:59-68.

Bradford JM (1986) Penetrability. In: Klute A (Ed.) Methods of Soil Analisys - Physical and Mineralogical Methods. 2a ed. Madison, ASA/SSSA. p.463-478.

Busscher WJ (1990) Adjustment of flat-tipped penetrometer resistance data to a common water content. Transactions of the American Society of Agricultural Engineers, 33:519-524.

Cavalieri KMV, Tormena CA, Vidigal Filho PS, Gonçalves ACA \& Costa ACS (2006) Efeitos de sistemas de preparo nas propriedades físicas de um Latossolo Vermelho distrófico. Revista Brasileira de Ciência do Solo, 30:137-147.

Rev. Ceres, Viçosa, v. 63, n.6, p. 868-878, nov/dez, 2016 
Collares GL, Reinert DJ, Reichert JM \& Kaiser DR (2006) Qualidade física do solo na produtividade da cultura do feijoeiro num Argissolo. Pesquisa Agropecuária Brasileira, 41:1663-1674.

Corrêa MM, Ker JC, Barrón V, Torrent J, Curi N \& Torres TCP (2008) Caracterização física, química, mineralógica e micromorfológica de horizontes coesos e fragipãs de solos vermelhos e amarelos do ambiente tabuleiros costeiros. Revista Brasileira de Ciência do Solo, 32:297-313.

Dourado Neto D, Nielsen DR, Hopmans JW, Reichardt K, Bacchi OOS \& Lopes PP (2001) Soil Water Retention Curve. version 3.00 beta. Piracicaba, Universidade de São Paulo.

Embrapa - Empresa Brasileira de Pesquisa Agropecuária (1997) Centro Nacional de Pesquisa de Solos. Manual de métodos de análise de solo. Rio de Janeiro, Embrapa. 212p.

Embrapa - Empresa Brasileira de Pesquisa Agropecuária (1999) Centro Nacional de Pesquisas de Solo. Sistema brasileiro de classificação de solos. Rio de Janeiro, Embrapa. 412p.

Embrapa - Empresa Brasileira de Pesquisa Agropecuária (2011) Manual de Métodos de Análise de Solo. Dados eletrônicos. $2^{\mathrm{a}}$ ed. Rio de Janeiro, Embrapa Solos. 230p.

Farias LI (2012) Comportamento mecânico e hídrico de um argissolo amarelo de tabuleiros costeiros cultivado com canade-açúcar. Dissertação de Mestrado. Universidade Federal de Sergipe, São Cristóvão. $41 \mathrm{p}$

Grable AR \& Siemer EG (1968) Effects of bulk density, aggregate size, and soil water suction on oxygen diffusion, redox potential and elongation of corns roots. Soil Science Society of America, $32: 180-186$

Haise HR, Haas HJ \& Jensen LR (1955) Soil misture studies of some great plains soils. II. Field capacity as related tp $1 / 3$ atmosphere percentage, and "minimum point" as related to 15and 26- atmosphere percentage. Soil Science Society of America, $34: 20-25$

Hamblin AP (1985) The influence of soil structure on water movement, crop root growth and water uptake. Advances in Agronomy, 38:95-158.

Kaiser DR, Reinert DJ, Reichert JM, Collares GL \& Kunz M (2009) Intervalo hídrico ótimo no perfil explorado pelas raízes de feijoeiro em um Latossolo sob diferentes níveis de compactação. Revista Brasileira de Ciência do Solo, 33:845-855.

Kay BD (1989) Rates of changes of soil struture under diferente cropping systems. Advances in Soil Science, 12:01-51.

Leão TP \& Silva AP (2004) A simmplified excel algarithm for estimating the least limiting water range of soils. Scientia Agricola, 61:649-654

Leão TP, Silva AP, Perfect E \& Tormena CA (2005) An algorithm for calculating the least limiting water range of soils. Agronomy Journal, 97:1210-1215.

Leão TP, Silva AP, Macedo MCM, Imhoff S \& Euclides VPB (2006) Least limiting water range: a potential indicator of changes in near-surface soil physical quality after the conversion of Brazilian Savanna into pasture. Soil \& Tillage Research, $88: 279-285$

Letey J (1985) Relationship between soil physical properties and crop productions. Advances in Soil Science, 1:277-294.

Lima Neto J de A, Ribeiro MR, Corrêa MM, Souza-Júnior VS de, Araújo Filho JC de \& Lima JFWF (2010) Atributos químicos, mineralógicos e micromorfológicos de horizontes coesos de latossolos e argissolos dos tabuleiros costeiros do estado de Alagoas. Revista Brasileira de Ciência do Solo, 34:473486.
Medeiros JC, Silva AP da, Cerri CEP, Giarola NFB, Figueiredo GC \& Fraceto FJC (2011) Linking physical quality and $\mathrm{CO}_{2}$ emissions under long-term no till and conventional-till in a subtropical soil in Brazil. Plant and soil, 338:05-15.

Melo Filho JF, Demattê JAM, Libardi PL \& Portela JC (2004) Comportamento espectral de um latossolo amarelo coeso argissólico em função de seu uso e manejo. Magistra, 16:105-112.

Moreau AMSS, Ker JC, Costa LM \& Gomes FH (2006) Caracterização de solos de duas topossequências em tabuleiros costeiros do sul da Bahia. Revista Brasileira de Ciência do Solo, 30:10071019.

Pereira PV, Ortiz-Escobar ME, Rocha GC, Assis Junior RN \& Oliveira TS (2012) Evaluation of soil physical quality of irrigated agroecosystems in a semi-arid region of North-eastern Brazil. Soil Research, 50:455-464

Pereira PV(2010) Avaliação da qualidade física de solos em áreas irrigadas na Chapada do Apodi, Ceará. Tese de Doutorado. Universidade Federal do Ceará, Fortaleza. 57p.

Reichert JM, Reinet DJ \& Braida JA (2003) Manejo, qualidade do solo e sustentabilidade: condições físicas do solo agrícola. $29^{\circ}$ Congresso Brasileiro de Ciência do Solo, Ribeirão Preto. Anais, SBCS. CD-ROM.

Reinert DJ, Reichert JM \& Silva VR (2001) Propriedades físicas de solos em sistema de plantio direto irrigado. In: Carlesso R, Petry MT, Rosa GM \& Ceretta CA (Eds.) Irrigação por aspersão no Rio Grande do Sul. Santa Maria, Universidade Federal de Santa Maria.165p.

Rezende JO (2000) Solos coesos dos tabuleiros costeiros: limitações agrícolas e manejo. Salvador, SEAGRI/SPA. 117p. (Série Estudos Agrícolas, 1)

Richards LA \& Weaver LR (1944) Fifteen atmosphere percentage as related to the permanent wiltin point. Soil Science, 56:331339.

Savage MJ, Ritchie JT, Bland WL \& Dugas WA (1996) Lower limit of soil water availability. Agronomy Journal, 88:844-651.

Silva FBR, Riché GR, Tonneau JP, Sousa Neto NC, Brito LTL, Correia RC, Cavalcanti AC, Silva FHBB, Silva AB \& Araújo Filho JC (1993) Zoneamento Ágroecológico do Nordeste: Diagnostico do quadro natural e agrossocioeconômico. Petrolina, Embrapa CPATSA / Recife, Embrapa CNPS. 387p.

Silva AP da, Kay BD \& Perfect E (1994) Characterization of the least limiting water range. Soil Science Society of America Journal, 58:1775-1781.

Silva AP \& Kay BD (1996) The sensitivity of shoot grought of corn to the least limiting water range of soils. Plant Soil, 184:323-329.

Silva AP da \& Kay BD (1997) Estimating the least limiting water range of soil from properties and management. Soil Science Society of America Journal, 61:877-883.

Silva AP, Tormena CA, Fidalski J \& Imhoff S (2008) Funções de pedotransferência para as curvas de retenção de água e de resistência do solo à penetração. Revista Brasileira de Ciência do Solo, 32:01-10.

Silva AP, Tormena CA, Imhoff SC, Fidalski J \& Neves Júnior A (2009) Intervalo hídrico ótimo e sua importância para as plantas. In: Ribeiro MR, Nascimento CW, Ribeiro Filho MR \& Cantalice JRB (Eds.) Tópicos em ciência do solo. Viçosa, Sociedade Brasileira de Ciência do Solo. p.01-30.

Silva GL, Lima HV, Campanha MM, Gilkes RJ \& Oliveira TS (2011) Soil physical quality of Luvisols under agroforestry, natural vegetation and conventional crop management systems in the Brazilian semi-arid region. Geoderma, 167:61-70. 
Souza ALV (2005) Avaliação da qualidade de um Latossolo Amarelo Coeso argissólico dos Tabuleiros Costeiros, sob floresta natural. Dissertação de Mestrado. Universidade Federal da Bahia, Cruz das Almas. 95p.

Souza LS (1996) Uso e manejo dos solos coesos dos tabuleiros costeiros. In: Reunião Técnica Sobre Solos Coesos Dos Tabuleiros, Cruz das Almas. Anais, EAUFBA/GVFBA, Embrapa/CNPMF. p. 36-75.

Taylor HM, Roberson GM \& Parker Jr. JJ (1966) Soil strengthroot penetration relations to medium to coarsetextured soil materials. Soil Science, 102:18-22.

Topp GC, Galganov YT, Wires KC \& Culley JLB (1994) Non limiting water range (NLWR): an approach for assessing soil structure. Ottawa, Agriculture and Agri-Food Canada. 36p. (Soil Quality Evaluation Programam, Report 2).
Tormena CA, Silva AP da \& Libardi PL (1998) Caracterização do intervalo hídrico ótimo de um Latossolo Roxo sob plantio direto. Revista Brasileira de Ciência do Solo, 22:573-581.

Tormena CA, Silva AP \& Libardi PL (1999) Soil physical quality of a Brazilian Oxisol under two tillage systems using the least limiting water range approach. Soil Tillage Research, 52:223232 .

Tormena CA, Silva AP, Imhoff SDC \& Dexter AR (2008) Quantification of the soil physical quality of a tropical Oxisol using the $\mathrm{S}$ index. Scientia Agricola, 65:56-60.

Van Genuchten MT (1980) A closed form equation for predicting the hydraulic conductivity of unsaturated soils. Soil Science Society of American journal, 44:892-898. 